\title{
Observation of Simultaneous Oscillation of Multiple Modes in a CW 300 GHz Gyrotron
}

\author{
Teruo SAITO, Tomoaki NAKANO, Seitaro MITSUDO, Isamu OGAWA and Toshitaka IDEHARA \\ Research Center for Development of Far-Infrared Region, University of Fukui, Fukui 910-8507, Japan
}

(Received 17 April 2007 / Accepted 2 May 2007)

\begin{abstract}
Multi-mode oscillation was observed in a $300 \mathrm{GHz}$ fully $\mathrm{CW}$ gyrotron. It has been developed and installed in the Research Center for Development of Far-Infrared Region, University of Fukui as a power source of a submillimeter-wave material processing system. This gyrotron delivers $1.75 \mathrm{~kW} / \mathrm{CW}$ at maximum. The radiation pattern is a Gaussian beam when the magnetic field strength $B_{\mathrm{c}}$ at the cavity is properly adjusted. However, within a range of $B_{\mathrm{c}}$ values, simultaneous oscillation of competing modes is observed, manifesting in radiation of the output power in multiple directions.
\end{abstract}

(C) 2007 The Japan Society of Plasma Science and Nuclear Fusion Research

Keywords: submillimeter CW gyrotron, radiation pattern, Gaussian beam, multi mode oscillation, material processing

DOI: $10.1585 /$ pfr.2.024

Medium-power millimeter/submillimeter waves have numerous applications in various fields. Among those applications, ceramic sintering and material processing are most promising. Recently, since a strong non-thermal effect is expected for higher frequency power, use of 20 - $30 \mathrm{GHz}$ gyrotrons has become popular [1]. A CW gyrotron with a frequency of hundreds of $\mathrm{GHz}$ has not yet been realized as a power source for practical applications, even though a stronger non-thermal effect can be expected. Thus, realization of a gyrotron with this frequency range and $\mathrm{kW}$ order power is strongly desired.

Mode competition is a serious problem in the design and operation of a medium to high power, high frequency gyrotron because the mode density inevitably becomes very high owing to the extremely oversized cavity. Usually, the designated mode with the smallest starting current grows by suppressing competing modes until finally, single-mode oscillation is realized.

However, there is a chance of multi-mode oscillation depending on the operation condition. We have observed this phenomenon on a fully $\mathrm{CW} 300 \mathrm{GHz}$ gyrotron called FU CW I. This is very interesting from the viewpoint of the gyrotron physics. Moreover, understanding of multi-mode oscillation is a basis for the development of medium-power $\mathrm{CW}$ gyrotrons with further higher frequencies.

This gyrotron with $\mathrm{kW}$ order output power has been developed according to the above requirements and installed in the Research Center for Development of FarInfrared Region, University of Fukui. It is the first gyrotron having these parameters for practical applications [2]. FU CW I works at the fundamental electron cyclotron resonance in a $12 \mathrm{~T}$ liquid helium-free superconducting magnet. It is operated at a relatively low cathode voltage of

author'se-mail: saitot@fir.fukui-u.ac.jp
$15 \mathrm{kV}$. The maximum beam current is $1 \mathrm{~A}$.

The designated cavity mode of FU CW I is $\mathrm{TE}_{22,8}$. The radius of the flat region of the cavity $R_{\mathrm{c}}$ is $8.39 \mathrm{~mm}$, and the resonance frequency of the $\mathrm{TE}_{22,8}$ mode is almost $300 \mathrm{GHz}$. The length of the flat region of the cavity is $15 \mathrm{~mm}$ and the total $\mathrm{Q}$ value including the Ohmic loss is about 6000 [3]. The gyrotron is equipped with a mode converter composed of a Vlasov-type radiator and three beamshaping mirrors. The output power is delivered through the vacuum window in a Gaussian beam. The vacuum window is made of $\mathrm{BN}$ and its diameter is $80 \mathrm{~mm}$. This gyrotron can be operated in either $\mathrm{CW}$ mode or pulse mode.

A performance test of FU CW I has been carried out in advance of its commissioning in a material processing system. A maximum $\mathrm{CW}$ output power of $1.75 \mathrm{~kW}$ was attained. This power was measured with a water load attached directly to the vacuum window. The radiation pattern was measured with an infrared camera. A Gaussian beam with a very low-level side lobe was confirmed when the magnetic field strength $B_{\mathrm{c}}$ at the cavity was properly adjusted. The oscillation was identified as the designated $\mathrm{TE}_{22,8}$ mode from frequency measurements performed by a combination of a harmonic mixer and a local oscillator [4].

Mode competition was observed in the range of the parameter space of $B_{\mathrm{c}}$ and the cathode voltage $V_{\mathrm{c}}$. Several modes oscillated simultaneously. This manifested in radiation of the output power into multi directions. Then the radiation pattern was measured in detail. A 1-mm-thick polyvinyl chloride plate was used as an absorber. Because of very low thermal conductivity of polyvinyl chloride, thermal diffusion was negligible in a few tens of seconds necessary for the temperature measurement. Therefore, the profile of temperature rise was considered to well represent the radiation pattern. 


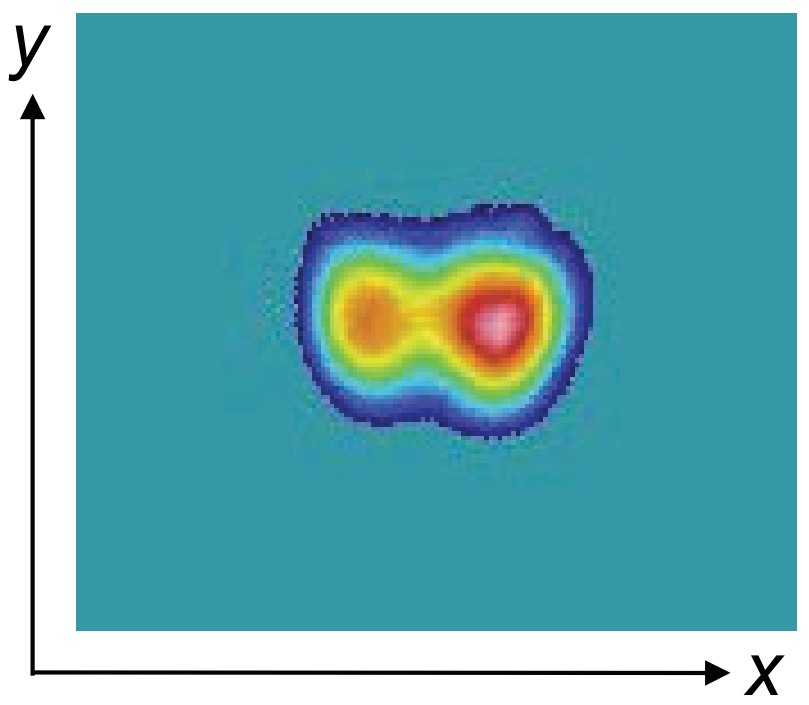

Fig. 1 An example of the infrared camera image for $V_{\mathrm{c}}=15 \mathrm{kV}$ and $B_{\mathrm{c}}=10.90 \mathrm{~T}$. The distance between the two peaks is about $3 \mathrm{~cm}$.

Figure 1 represents a typical temperature profile for the case of two peaks. In this case, $V_{\mathrm{c}}$ was $15 \mathrm{kV}$ and the beam current $I_{\mathrm{b}}$ was about $1 \mathrm{~A}$. The multi-peak radiation pattern depends sensitively on $B_{\mathrm{c}}$ and $V_{\mathrm{c}}$. For $V_{\mathrm{c}} \leq 12 \mathrm{kV}$, single-peak radiation is observed within almost all ranges of $B_{\mathrm{c}}$.

The multi-peak character also depends on $B_{\mathrm{c}}$. Figure 2 shows the temperature distribution of the absorber along the horizontal direction (along the $x$ direction of Fig. 1) for different $B_{\mathrm{c}}$ values, with $V_{\mathrm{c}}$ fixed at $15 \mathrm{kV}$. Multi peaks always appear along the horizontal direction, and the position corresponding to each peak does not vary for different values of $B_{\mathrm{c}}$. Therefore, each peak is considered to correspond to a different oscillation mode. As shown by the red line in Fig. 2, only one beam is radiated when $B_{\mathrm{c}}$ is set at $10.95 \mathrm{~T}$. This beam is radiated in the same direction as that for $V_{\mathrm{c}}$ of $12 \mathrm{kV}$, for which only one beam is radiated within a wide range of $B_{\mathrm{c}}$ values. This oscillation was identified as the $\mathrm{TE}_{22,8}$ mode from the frequency measurement. Thus, the radiation shown by the red line in Fig. 2 originates from the $\mathrm{TE}_{22,8}$ mode. We refer to this beam as Beam 1.

The peak temperature of the absorber corresponding to the power of Beam 1 decreased with decreasing $B_{\mathrm{c}}$. A new peak appeared in the position left of the Beam 1 peak when $B_{\mathrm{c}}$ was slightly decreased, as depicted by the black line in Fig. 2. We call it Beam 2. The output power of the oscillation corresponding to this peak was rather low and disappeared for smaller $B_{\mathrm{c}}$ values. Instead, another peak turned up in between Beam 1 and Beam 2. The green line in Fig. 2 represents this case. This peak is called Beam 3. When $B_{\mathrm{c}}$ was further decreased, Beam 1 disappeared and Beam 3 alone was observed as shown by the blue line in Fig. 2. The difference in $B_{\mathrm{c}}$ between the case of the red line

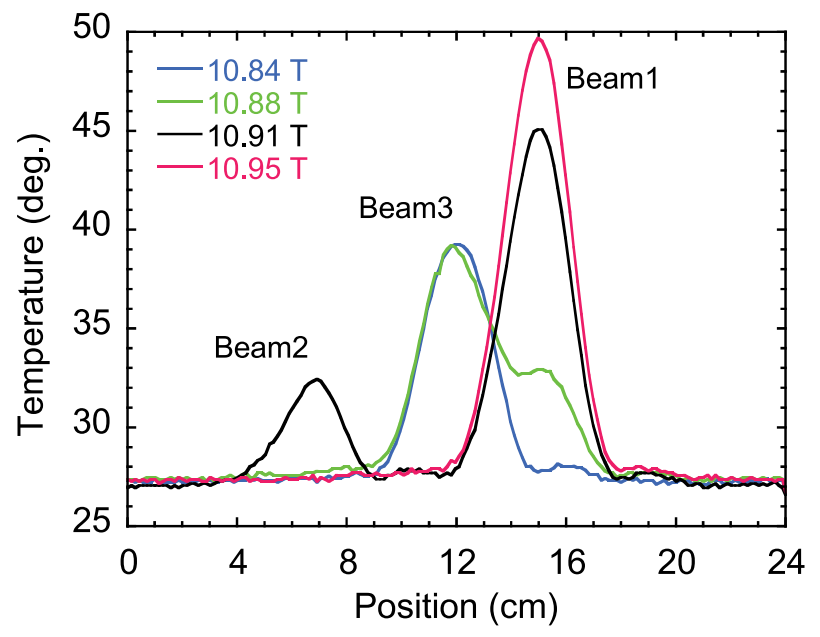

Fig. 2 Temperature distributions along the horizontal line that passes the peak temperature position.

and the case of the blue line was $0.11 \mathrm{~T}$.

In addition to the $\mathrm{TE}_{22,8}$ mode, a $\mathrm{TE}_{19,9}$ mode was also identified from the frequency measurement. The difference of $0.11 \mathrm{~T}$ in $B_{\mathrm{c}}$ just corresponds to that between the optimum values of $B_{\mathrm{c}}$ for both modes. Therefore, Beam 3 is considered to originate from the $\mathrm{TE}_{19,9}$ mode. The counter-rotating $\mathrm{TE}_{19,9}$ mode is the most dangerous competing mode. The oscillation mode corresponding to Beam 2 has not been identified yet.

The $\mathrm{TE}_{22,8}$ mode and the $\mathrm{TE}_{19,9}$ mode simultaneously oscillate in a range of $B_{\mathrm{c}}$ values when $V_{\mathrm{c}}$ is larger than $13 \mathrm{kV}$. When the beam current $I_{\mathrm{b}}$ is decreased, the oscillation range of $B_{\mathrm{c}}$ becomes slightly narrower for each mode. Finally, the multi-oscillation region disappears for $I_{\mathrm{b}}$ smaller than $0.3 \mathrm{~A}$.

The radiation pattern was measured in the pulse mode to avoid burn-up of the absorber, but the infrared camera recorded the temperature profile on the absorber plate as a result of time integration of the absorbed power. Therefore, the multi-peak pattern does not directly indicate simultaneous oscillation of the multi modes. For this reason, pyroelectric detectors were set on the lines of Beam 1 and 3 . The signals of the two detectors clearly showed simultaneous and full-time oscillation of the two modes during the cathode voltage pulse. For each beam, the dependence of the signal intensity on $B_{\mathrm{c}}$ is similar to the dependence of the temperature rise on $B_{\mathrm{c}}$.

Simultaneous oscillation of multi modes was observed in a $140 \mathrm{GHz}$ gyrotron [5]. The main operation mode of this gyrotron was relatively low-mode $\mathrm{TE}_{03}$ at the fundamental resonance. Competition between the fundamental and the second harmonic oscillations was also reported [6]. The frequency of the second harmonic oscillation was $383 \mathrm{GHz}$. Compared with these experiments, the oscillation modes of FU CW I are very high.

Another problem is how the counter-rotating mode 
can be radiated as a Gaussian beam through the internal mode converter of the Vlasov type. The co-rotating $\mathrm{TE}_{19,9}$ mode could be excited, but the optimum radius of the electron beam is considerably smaller than that of the counterrotating $\mathrm{TE}_{19,9}$ mode. We have not come to the physical understanding of this phenomenon yet. Results of more detailed analysis of experimental data and theoretical discussion will be published elsewhere.

[1] H. Hoshizuki et al., Int. J. Infrared Millimeter Waves 26,
1531 (2005).

[2] S. Mitsudo et al., Conf. Digest of 31st IRMMW E 14th THz (2006) p.572.

[3] V.E. Zapevalov et al., Conf. Digest of 29th IRMMW E 12th $\mathrm{THz}$ (2004) p.149.

[4] T. Saito et al., Conf. Digest of 31st IRMMW $\mathcal{E}$ 14th THz (2006) p.24.

[5] K.E. Kreischer et al., IEEE Trans. Microwave Theory Tech. MTT-32, 481 (1984).

[6] T. Idehara et al., Phys. Fluids B 4, 267 (1992). 